Revista Brasileira de

Engenharia Agrícola e Ambiental

v.16, n.3, p.268-274, 2012

Campina Grande, PB, UAEA/UFCG - http://www.agriambi.com.br

agriambi

Protocolo 115.11 - 04/06/2011 • Aprovado em 29/12/2011

\title{
Manejo da irrigação utilizando sensor da umidade do solo alternativo ${ }^{1}$
}

\author{
Wellington A. de Freitas ${ }^{2}$, Jacinto de A. Carvalho', \\ R. A. Braga ${ }^{2} \&$ Messias J. B. de Andrade ${ }^{3}$
}

\begin{abstract}
RESU M O
Um sensor alternativo utilizando a medida da capacitância para determinação da umidade do solo foi desenvolvido e calibrado em laboratório, e avaliado por meio da comparação com tensiômetros, no manejo da irrigação da cultura do feijoeiro. U tilizou-se um delineamento inteiramente casualizado com 5 repetições e esquema fatorial com dois métodos de manejo (alternativo e tensiometria) além tensões de água no solo $(20,40$ e $60 \mathrm{kPa})$. O bservou-se que, independente do método empregado, a produção do feijoeiro foi maior quando submetido a tensão de $39 \mathrm{kPa}$; entretanto, o manejo da irrigação utilizandose o método alternativo levou a um número maior de irrigações e também do volume de água aplicado, com consequente redução da eficiência do uso da água em comparação com o uso de tensiômetros.
\end{abstract}

Palavras-chave: capacitância, feijão, tensão de água no solo

\section{Irrigation scheduling using alternative soil moisture sensor}

\begin{abstract}
An alternative sensor using a capacitance measurement for determining soil moisture was developed and calibrated in the laboratory and evaluated by comparison with tensiometers in the irrigation of common bean. A completely randomized design was used with five replications and a factorial with two methods of management (alternative and tensiometer) and soil water tensions (20, 40 and $60 \mathrm{kPa})$. It was observed that regardless of the method, the bean yield was higher when subjected to stress of $39 \mathrm{kPa}$, however, irrigation management using the alternative method led to a greater number of irrigation and al so the volume of water applied, with consequent reduction in water use efficiency compared to the use of tensiometers.
\end{abstract}

Key words: capacitance, common bean, soil water tension

\footnotetext{
1 Parte da Dissertação do primeiro autor

2 Departamento de Engenharia/U FLA, CP 3037, CEP 37200-000, Lavras, M G. Fone: (35) 3829-1489. E-mail: freitao@hotmail.com; jacintoc@deg.ufla.br; robbraga@deg.ufla.br

${ }^{3}$ D epartamento de Agricultura/U FLA. Fone (35) 3829-1327. E-mail: mandrade@dag.ufla.br
} 


\section{INTRODUÇÃO}

Diversos são os métodos de determinação da umidade do solo. Atualmente, os métodos eletrométricos vêm ganhando espaço em função de sua maior versatilidade. Segundo Conklin (2005) medidas que envolvem eletricidade (voltagem, corrente, frequência, resistência, capacitância, propriedades dielétricas) podem ser utilizadas individualmente ou em combinação para obter informações sobre o meio no qual os elétrons estão se movendo.

Pesquisas vêm sendo realizadas acerca dos parâmetros supracitados com objetivo de desenvolver sensores capazes de monitorar as variáveis do ambiente agrícola. Celinski \& Zimback (2010) desenvolveram um sensor de capacitância elétrica e avaliaram seu desempenho em campo. Segundo os autores, alguns atributos do solo ( $\mathrm{pH}, \mathrm{CTC}$, soma de base, cálcio, magnésio) se correlacionaram significativamente com a capacitância, demonstrando a possibilidade de ser utilizado no controle da fertilidade do solo.

O conhecimento da constante dielétrica dos materiais é um dos princípios básicos no desenvolvimento de sensores capacitivos. Esses sensores têm sido empregados para determinar a umidade do solo, devido ao valor da constante dielétrica da água. Segundo alguns autores, Rusiniak (2002), Chang et al. (2007), Kumhála et al. (2008), Frangi et al. (2009), Benedetto (2010) e Monsen-Nia et al. (2010), este valor é de 7880 , em contraste com o solo, cujo valor pode chegar a até 14 . Assim, a constante dielétrica é altamente relacionada com o teor de água no solo (Kizito et al., 2008).

Medir o teor de água do solo pela determinação da capacitância não é uma idéia nova, tendo como exemplos os trabalhos desenvolvidos por Anderson \& Edlefsen (1942), Anderson (1943) e Plater (1955). A partir dos anos 60 pesquisas sobre o comportamento dielétrico dos líquidos polares e sólidos tiveram maior notoriedade (Williams, 2011). Entretanto, somente a partir das últimas décadas esta técnica vem sendo explorada de maneira expressiva em virtude principalmente do desenvolvimento de instrumentos eletrônicos mais sofisticados e com aplicações na agricultura irrigada como, por exemplo, os trabalhos conduzidos por Freitas et al. (2009) e Cruz et al. (2010).

A criação e o desenvolvimento de novos métodos para estimativa de parâmetros utilizados no manejo da irrigação, poderão contribuir para obtenção de maiores eficiências dos processos e menores custos. Objetivou-se, com o presente trabalho, o desenvolvimento, a calibração e avaliação de sensores alternativos (capacitores) para monitorar a umidade do solo.

\section{Material e MÉTOdos}

Os tensiômetros (TS) e sensores alternativos (SA) utilizados no experimento foram construídos e testados no Laboratório de Hidráulica do DEG/UFLA. Para construção dos TS utilizaram-se tubos de PVC branco de $12 \mathrm{~mm}$, cápsulas cerâmicas, tubos de acrílico $8 \mathrm{~mm}$ de diâmetro, rolha de borracha e cola epóxi. Antes de se realizar os testes os tensiômetros permaneceram submersos em água, durante $24 \mathrm{~h}$, para saturação das cápsulas; após este período, eles foram colocados dentro de recipiente contendo água e submetidos a uma pressão interna de $150 \mathrm{kPa}$, através de um compressor. Tal procedimento teve como objetivo identificar possíveis vazamentos.

Para a construção dos SA foram utilizados tubos de cobre, solda à base de estanho, fio paralelo de $1 \mathrm{~mm}^{2}$, forma de alumínio e, como material dielétrico, gesso, devido à sua característica higroscópica, fácil moldagem e preço acessível. Utilizando cortador específico, realizou-se o corte dos tubos de cobre e de PVC nas dimensões adotadas (12 e $14 \mathrm{~mm}$ ) respectivamente; em seguida, o eletrodo de $22 \mathrm{~mm}$ de diâmetro foi ajustado no interior do tubo de PVC, para não haver contato do eletrodo com o solo.

A etapa seguinte consistiu em soldar um terminal do fio no interior do eletrodo. Na sequência, esse conjunto foi colocado na forma de alumínio para preenchimento com a mistura água/ gesso; após $5 \mathrm{~min}$ o eletrodo de $15 \mathrm{~mm}$ de diâmetro foi inserido no interior do eletrodo de $22 \mathrm{~mm}$ de maneira concêntrica. Após secar ao ar, esse conjunto foi retirado para soldagem do outro terminal no eletrodo menor. Terminada a construção dos SA, prosseguiu-se com a calibração. Na Figura 1 se vislumbra, de maneira esquemática, o procedimento descrito.

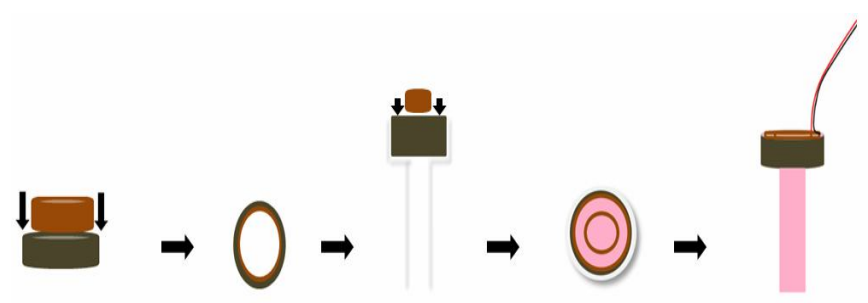

Figura 1. Etapas da construção dos sensores alternativos

Para mensurar a capacitância utilizou-se um capacímetro digital da marca ICEL, modelo CD - 300. A tensão máxima de saída nos terminais é de $2,8 \mathrm{~V}_{\mathrm{RMS}}$, a frequência do sinal aplicado varia conforme a escala do aparelho, no experimento a escala utilizada foi $2000 \mu \mathrm{F}$ com resolução de $1 \mu \mathrm{F}$ e frequência de 8 $\mathrm{Hz}$, segundo manual do fabricante.

O processo de calibração consistiu em saturar quatro SA utilizando-se água destilada, durante $24 \mathrm{~h}$; prosseguindo, os mesmos foram submetidos a diferentes valores de pressão, para cujo procedimento utilizou-se um sistema pressurizado com mangueiras de PVC, as quais estavam conectadas a um compressor para forçar a retirada de água dos SA.

Para cada valor de pressão aplicada (20, 50, 100, 150 e 200 $\mathrm{kPa}$ ), a capacitância foi determinada submetendo-se cada capacitor a um tempo de carga correspondente a um minuto. Para o monitoramento da pressão utilizou-se um manômetro digital da marca GULTON, modelo GULpress 1000. Os SA permaneceram dentro das cápsulas, sob pressão, durante $90 \mathrm{~h}$. A partir deste momento, correlacionou-se tensão de água no dielétrico com o valor médio da capacitância. Para avaliação dos sensores alternativos os mesmos foram utilizados em experimento com manejo da irrigação da cultura do feijoeiro. 
O experimento foi conduzido em casa de vegetação do tipo pavilhão, na Universidade Federal de Lavras, na cidade de Lavras, Minas Gerais, situado a $918 \mathrm{~m}$ de altitude, coordenadas geográficas $21^{\circ} 14^{\prime}$ de Latitude Sul e $45^{\circ} 00^{\prime}$ de Longitude de Greenwich. De acordo com a classificação Koppen, a região apresenta clima Cwa, clima temperado suave, chuvoso, com inverno seco, temperatura média do mês mais frio inferior a $18{ }^{\circ} \mathrm{C}$ e superior a $3{ }^{\circ} \mathrm{C}$, o verão apresenta temperatura média do mês mais quente, superior a $22{ }^{\circ} \mathrm{C}$ (Dantas et al., 2007).

Os valores de temperatura e umidade relativa do ar dentro da casa de vegetação, foram monitoradas diariamente através da leitura de um termohigrômetro digital; tais leituras foram realizadas diariamente, às $8 \mathrm{~h}$. $\mathrm{O}$ experimento foi conduzido com delineamento inteiramente casualizado (DIC), com 5 repetições e esquema fatorial, sendo $2 \times 3$ tratamentos (capacitores e tensiômetros) nas tensões de 20, 40 e 60 kPa, totalizando 30 parcelas experimentais.

O solo utilizado foi coletado no campus da UFLA, na camada subsuperficial $(0,10 \mathrm{~m}$ a $0,40 \mathrm{~m}$ de profundidade), classificado como Latossolo Vermelho Distroférrico segundo novo sistema hierarquizado (EMBRAPA, 2006) e submetido a tamisagem através de peneira com malha de $5 \mathrm{~mm}$; a partir de então, realizouse a coleta de uma amostra composta representativa para análises físicas e químicas, as quais foram realizadas nos Laboratórios de Física do Solo do Departamento de Ciência do Solo (DCS/UFLA). O solo utilizado foi classificado como argiloso.

Os parâmetros da curva de retenção foram ajustados através do aplicativo Solver do programa Microsoft Excel ${ }^{\circledR}$, com base no modelo de Genuchten (1980) mostrado na Eq. (1).

$$
\theta=0,4215 \times\left[1+\left(0,2040 \times\left|\Psi_{\mathrm{m}}\right|\right)^{1,8757}\right]^{-0,4669}+0,2670
$$

A adubação ocorreu 30 dias antes da semeadura, modelada nos resultados da análise de fertilidade do solo e de acordo com as recomendações da $5^{\text {a }}$ Aproximação da Comissão de Fertilidade do Solo do Estado de Minas Gerais (CFSEMG, 1999). Após esta etapa realizou-se o preenchimento dos vasos de polietileno de $18 \mathrm{dm}^{3}$ com solo.

Utilizou-se a cultivar BRSMG Majestoso, pertencente ao tipo carioca; após a distribuição dos vasos, montagem e teste do sistema de irrigação, foram semeadas com solo na capacidade de campo, cinco sementes em cada vaso. Decorridos 6 dias após semeadura (DAS), efetuou-se o desbaste deixando-se as 3 melhores plantas de cada vaso; até os 10 DAS todos os tratamentos foram irrigados igualmente; enfim se iniciou, após este período, o tratamento.

As plantas foram irrigadas utilizando-se sistema de gotejamento por gravidade com emissores autocompensantes, de vazão igual a 4,0 $\mathrm{L} \mathrm{h}^{-1}$. O manejo da irrigação foi realizado com base na leitura média de três capacitores e tensiômetros, instalados a $15 \mathrm{~cm}$ de profundidade nas unidades experimentais. A produção por planta ( $\mathrm{g}$ ) foi avaliada e os dados submetidos à análise de variância. As análises estatísticas foram realizadas utilizando-se o software SISVAR, versão 5.3 Build 75 (Ferreira, 2000).

\section{RESULTADOS E DISCUSSÃO}

Na Figura 2 são mostradas as variações dos valores de capacitância, em função do tempo de exposição, para cada valor de tensão estudado.

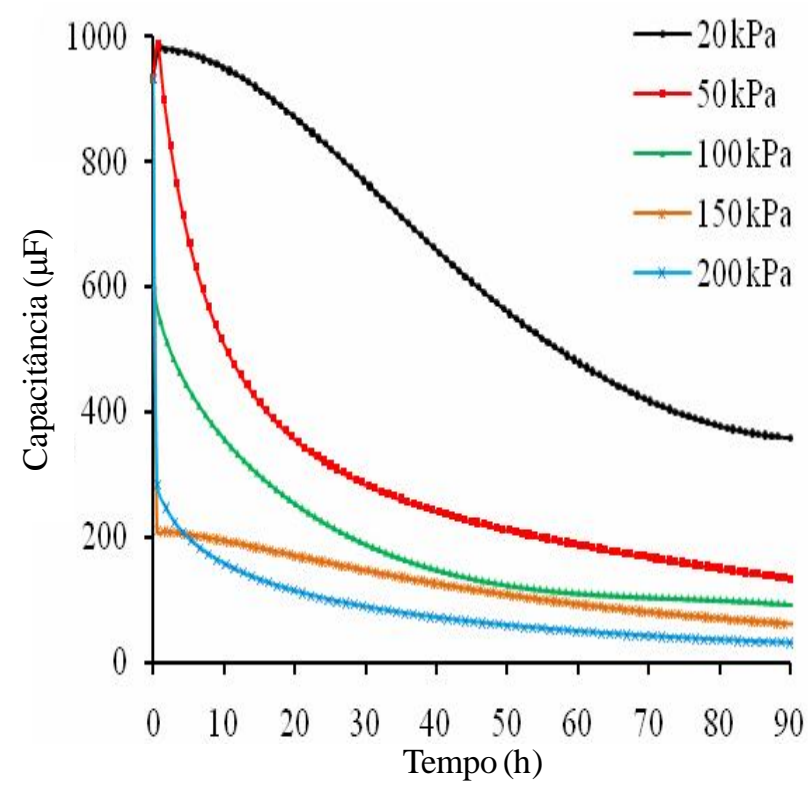

Figura 2. Comportamento da capacitância sob diferentes tensões

Observou-se, durante o processo de calibração, maior variação na capacitância quando submetida a tensões menores, principalmente a tensão de $20 \mathrm{kPa}$. Supõe-se que este fenômeno decorre da maior mobilidade dos íons presentes no dielétrico (sulfato de cálcio), uma vez que a resposta dielétrica de alguns materiais, em frequências abaixo do visível e do ultravioleta, é influenciada pela componente iônica (Benedetto, 2010). Na Figura 3 se encontram os valores da capacitância para o período de $90 \mathrm{~h}$.

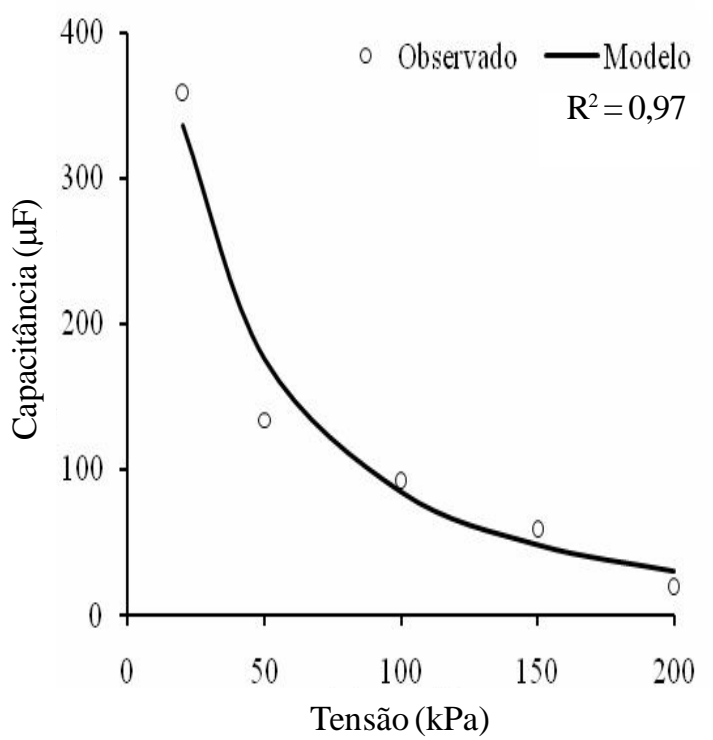

Figura 3. Correlação entre capacitância e tensão de água no bloco de gesso 
A partir dos valores obtidos durante o processo de calibração, ajustou-se a Eq. 2, a qual foi utilizada para realizar o manejo da irrigação.

$$
\text { Cap }=\mathrm{e}^{\left(6,9348-0,2498 \times \mathrm{T}^{0,5}\right)}
$$

em que:

$\mathrm{T}$ - tensão, $\mathrm{kPa}$;

Cap - capacitância, $\mu \mathrm{F}$

A temperatura e a umidade relativa do ar médias, ao longo do experimento, no interior da casa de vegetação foram, respectivamente, de $25,30{ }^{\circ} \mathrm{C} \mathrm{e} 61,34 \%$, valores dentro da faixa ideal para a cultura do feijoeiro, segundo Monteiro (2009).

Na Tabela 1 são apresentadas as análises de variância dos dados referentes à massa de grãos por planta $\left(\mathrm{M}_{\mathrm{GP}}\right)$, volume de água aplicado $\left(\mathrm{V}_{\mathrm{AP}}\right)$ e eficiência de uso da água (EUA).

Tabela 1. Resumo da análise de variância

\begin{tabular}{lrccc}
\hline \multirow{2}{*}{$\mathbf{F V}$} & \multirow{2}{*}{$\mathbf{G L}$} & \multicolumn{3}{c}{ Quadrado médio } \\
\cline { 3 - 5 } & & $\mathbf{M}_{\mathbf{G P}}(\mathbf{g})$ & $\mathbf{V}_{\mathrm{AP}}(\mathbf{L})$ & $\mathbf{E U A}\left(\mathbf{m g ~ L}^{-1}\right)$ \\
Método (M) & 1 & $3,30^{\text {ns }}$ & $3583,7284^{*}$ & $421025,79^{*}$ \\
Tensão (T) & 2 & $3,04^{*}$ & $63,5973^{*}$ & $279,36^{\text {ns }}$ \\
Interação (M x T) & 2 & $2,16^{\text {ns }}$ & $49,3199^{*}$ & $22112,34^{*}$ \\
Resíduo & 24 & 0,83 & 7,61 & 4454,81 \\
CV (\%) & - & 10,98 & 6,09 & 11,5 \\
Média geral & - & 8,28 & 45,297 & 580,3 \\
\hline
\end{tabular}

"Significativo a nível de $5 \%$ de probabilidade pelo teste $\mathrm{F}$; ns - não significativo pelo teste $\mathrm{F}$ a $5 \%$ de probabilidade; FV - fonte de variação; GL - grau de liberdade; CV - coeficiente de variação

Os resultados da Tabela 1 mostram que a massa de grãos por planta não foi influenciada pelos métodos de manejo da irrigação porém o volume de água aplicado e a eficiência de uso da água foram afetados significativamente pelos métodos. Na Figura 4 vê-se o comportamento da massa de grãos por planta, nas diferentes tensões.

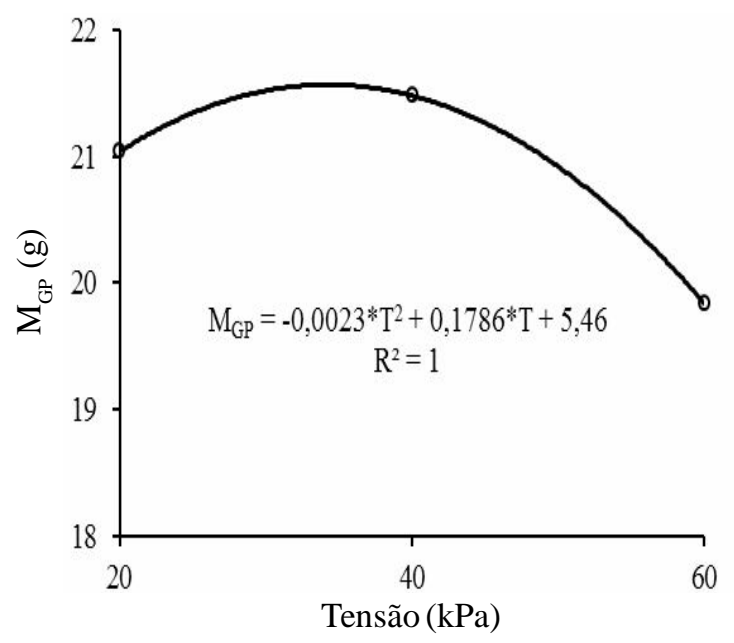

Figura 4. Relação entre massa de grãos planta-1 e tensão de água no solo

De acordo com a equação ajustada, a tensão ótima para a massa de grãos foi de 38,82 kPa. Queiroz (2004) ajustou, empregando tensiômetros com vacuômetro de Bourdon adaptados ao manejo da irrigação na cultura do feijoeiro (cultivar BRS Talismã), um modelo quadrático cujo ponto de máximo rendimento foi de $38,55 \mathrm{kPa}$.

Estudo realizado por Guimarães et al. (2006) demonstrou que o genótipo Carioca apresentou melhor capacidade de recuperação e manutenção de altos potenciais de água na folha que a linhagem RAB 96, quando submetido a déficit hídrico; entretanto, Paula Júnior et al. (2008), recomendam valores de tensão entre 10 a 40 $\mathrm{kPa}$, haja vista que representam condições ideais de água e arejamento do solo. Em concordância com esses autores, Lopes et al. (2011) e Sant'Ana et al. (2011) adotaram, estudando o efeito da aplicação de nitrogênio em feijoeiro irrigado, para fins de manejo de irrigação, a tensão limite de $40 \mathrm{kPa}$.

Na Tabela 2 se acham os valores médios do volume de água aplicado nos diferentes métodos de manejo, em cada tensão.

Tabela 2. Valor médio do volume de água aplicada $\mathrm{V}_{\mathrm{AP}}$ (L) em cada método e tensões

\begin{tabular}{cccc}
\hline \multirow{2}{*}{ Método $^{*}$} & \multicolumn{3}{c}{ Tensão (kPa) } \\
\cline { 2 - 4 } & $\mathbf{2 0}$ & $\mathbf{4 0}$ & $\mathbf{6 0}$ \\
$\mathrm{SA}$ & $58,17 \mathrm{Aa}$ & $55,92 \mathrm{Aa}$ & $54,58 \mathrm{Aa}$ \\
TS & $33,38 \mathrm{Ab}$ & $39,17 \mathrm{Bb}$ & $30,56 \mathrm{Ab}$ \\
\hline
\end{tabular}

As médias seguidas com a mesma letra minúscula na vertical e maiúscula na horizontal, não diferem estatisticamente entre si a nível de $5 \%$ de probabilidade, pelo teste Scott-Knott

* SA - sensor alternativoç TS - tensiômetro

Em todas as tensões observa-se que o volume de água aplicado foi maior ao se utilizar o SA em relação ao TS. Embora não tenha havido diferença de volume de água aplicada, utilizando-se o SA, o intervalo entre as irrigações no tratamento de $20 \mathrm{kPa}$ foi menor, ou seja, maior frequência de irrigação, ocorrendo drenagem. Verificou-se, ao longo do experimento, que os SA foram mais suscetíveis à variação de umidade do solo devido, provavelmente, ao maior tamanho dos poros do dielétrico em relação à cápsula porosa do tensiômetro.

Persson et al. (2006) comprovaram, desenvolvendo sen sor para determinação da tensão de água no solo com tecnologia TDR, que a distribuição do tamanho dos poros do material dielétrico interfere nos valores de tensão de água no solo. $\mathrm{Na}$ Tabela 3 são mostrados os valores médios da eficiência do uso de água (EUA) nos diferentes métodos de manejo e tensões.

Tabela 3. Valores médios da eficiência do uso de água $\left(\mathrm{mg} \mathrm{L}^{-1}\right)$ em cada méto do e tensões

\begin{tabular}{cccc}
\hline Método* $^{*}$ & \multicolumn{3}{c}{ Tensão (kPa) } \\
\cline { 2 - 4 } & $\mathbf{2 0}$ & $\mathbf{4 0}$ & $\mathbf{6 0}$ \\
SA & $410,26 \mathrm{Aa}$ & $496,50 \mathrm{Aa}$ & $478,73 \mathrm{Aa}$ \\
TS & $750,74 \mathrm{Ab}$ & $653,32 \mathrm{Ab}$ & $692,22 \mathrm{Ab}$ \\
\hline
\end{tabular}

As médias seguidas com a mesma letra minúscula na vertical e maiúscula na horizontal, não diferem estatisticamente entre si a nível de $5 \%$ de probabilidade, pelo teste Scott-Knott * SA - sensor alternativoç TS - tensiômetro

Os resultados da Tabela 3 evidenciam, para todas as tensões, que o manejo utilizando TS é mais eficiente. Conforme mencionado anteriormente, a porosidade do material dielétrico pode ter influenciado nesse resultado.

Na Figura 5 são mostrados os valores diários de tensão ao longo do ciclo da cultura para os respectivos métodos de manejo. A Figura 5A e 5B refere-se ao tratamento submetido à 
A.

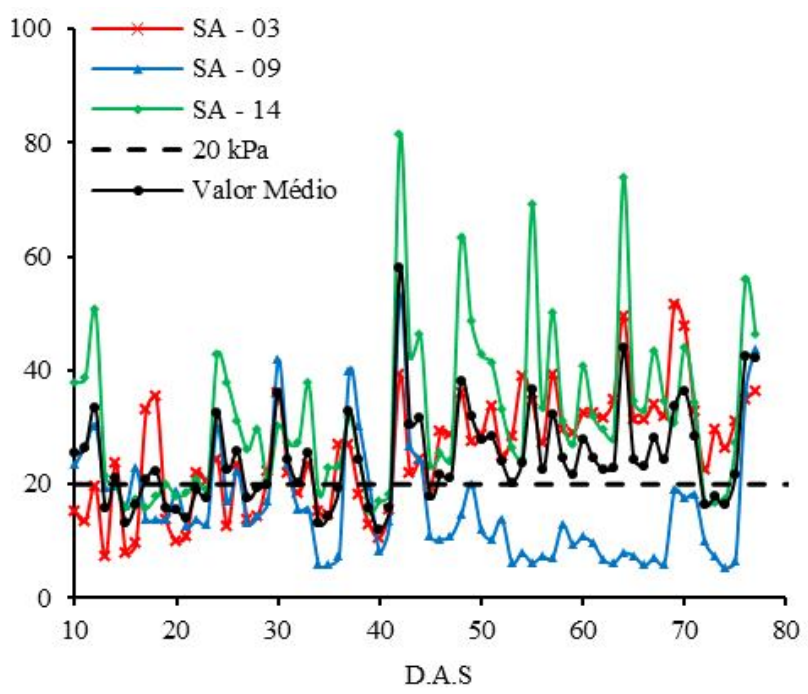

C.

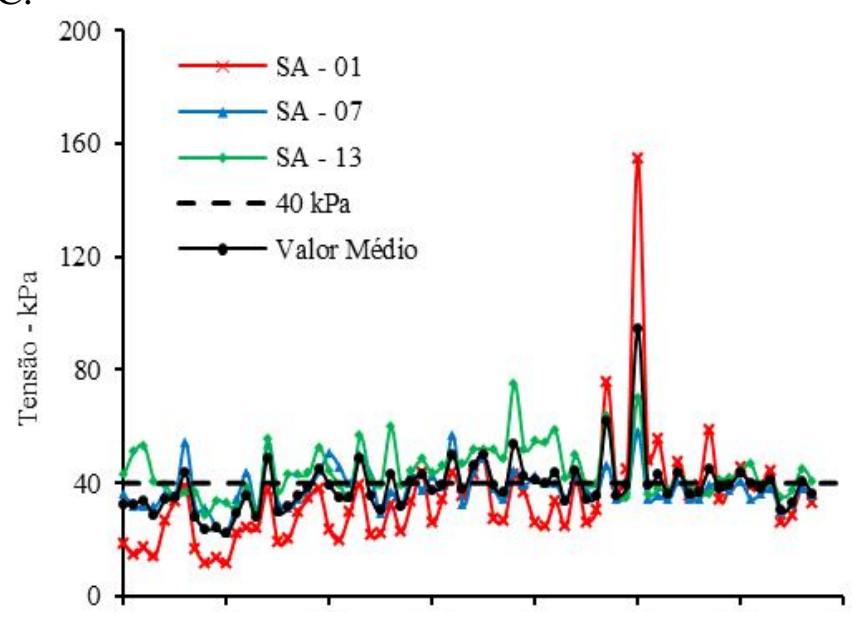

E.

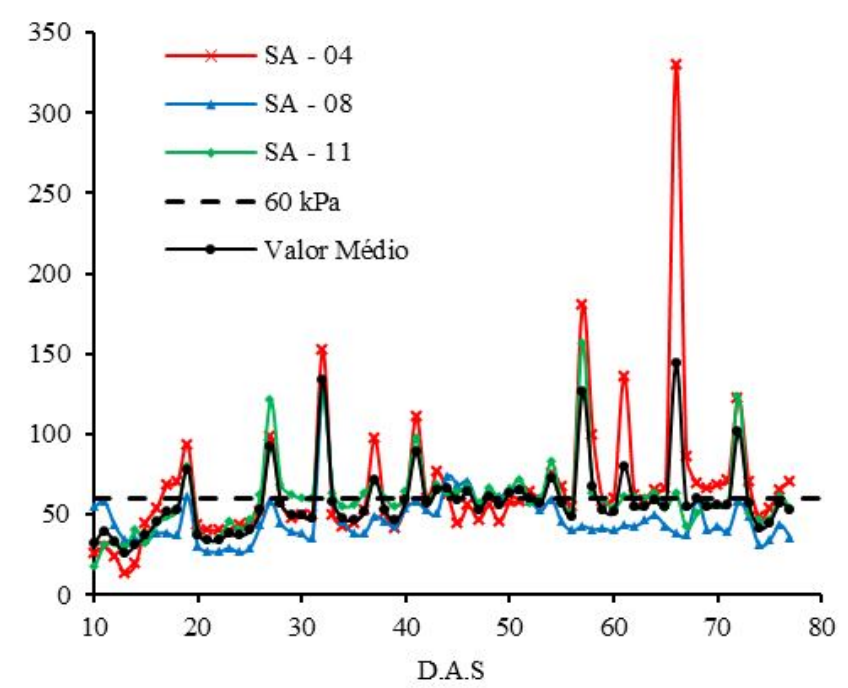

B.

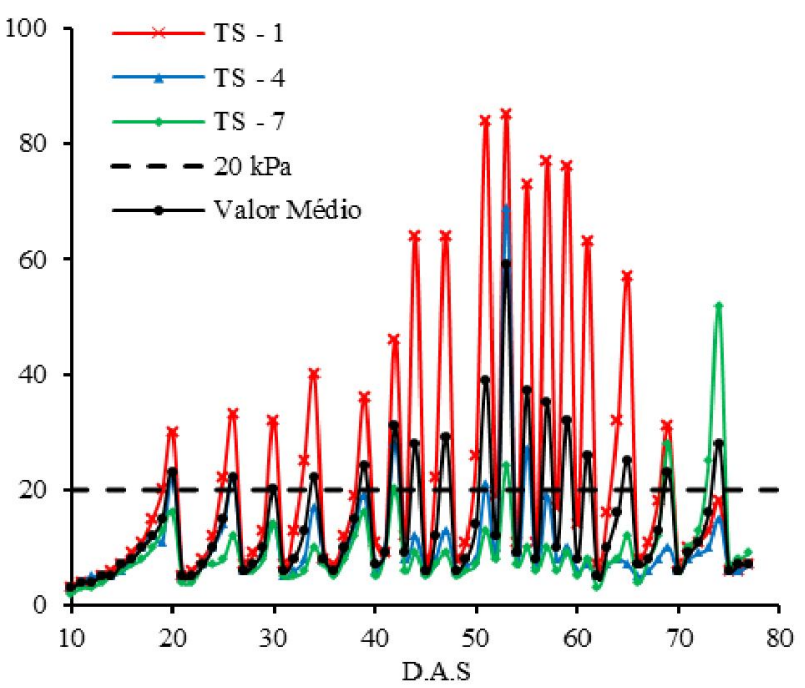

D.

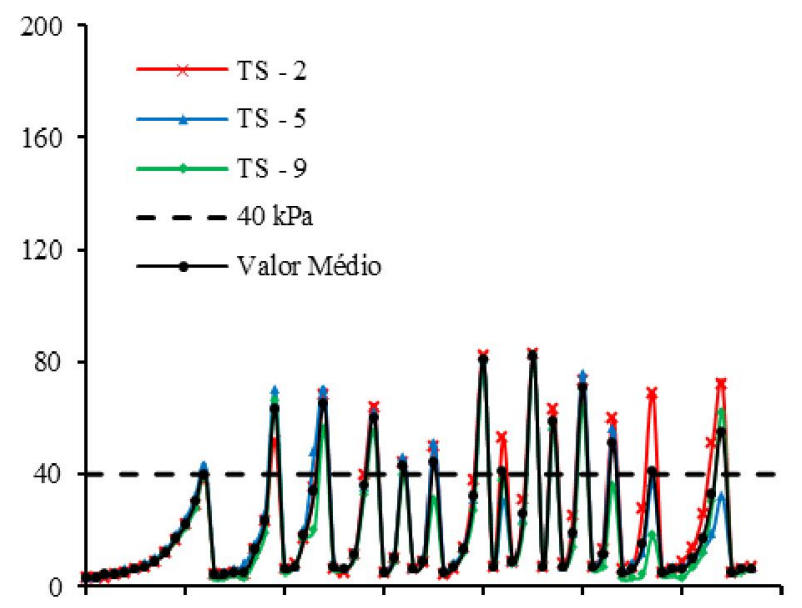

F.

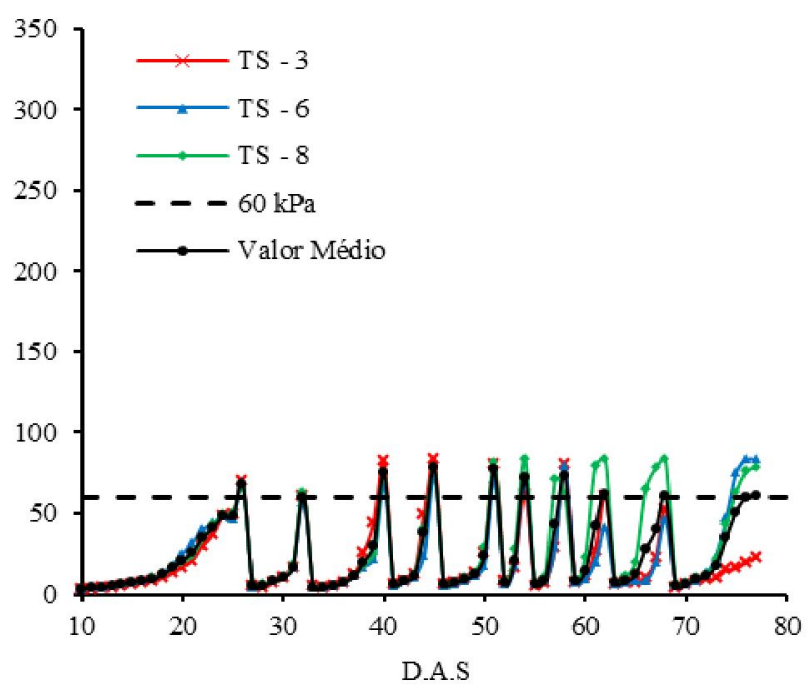

Figura 5. Comportamento dos SA e TS para diferentes tensões de água no solo em função dos dias após a semeadura (DAS)

tensão de $20 \mathrm{kPa}$, utilizando-se o SA e TS, respectivamente. A irrigação foi realizada toda vez em que os valores médios de tensão estavam acima da linha de referência.
O manejo de irrigação utilizando os SA, para tensão de 20 $\mathrm{kPa}$, apresentou variação considerável. É importante frisar que tensões menores (solo mais úmido) apresentaram maior variação 
nos valores de capacitância, durante o processo de calibração. Este fenômeno decorre, possivelmente, devido à maior mobilidade dos íons presentes na solução do solo ou mesmo dos íons presentes no dielétrico (sulfato de cálcio), uma vez que a resposta dielétrica de alguns materiais, em frequências abaixo do visível e do ultravioleta, é influenciada pela componente iônica (Benedetto, 2010).

O manejo da irrigação utilizando-se os TS, também apresentou variação, em que o comportamento pode ser devido à distribuição no tamanho de poros da cápsula cerâmica dos TS. O TS 1 (Figura 5B) apresentou relativamente maior variação durante boa parte do experimento, embora a partir dos 65 DAS essa variação tenha diminuído em virtude, da menor demanda de água por parte das plantas. Independente do método empregado no manejo observou-se variação nas medidas.

Nas Figuras 5C e 5D se encontram os valores de capacitância e tensão, respectivamente, no manejo de irrigação para tensão de $40 \mathrm{kPa}$. Nesse tratamento de $40 \mathrm{kPa}$, verifica-se uma variação menor entre as leituras, em ambos os métodos de manejo, em relação ao tratamento de $20 \mathrm{kPa}$.

Nas Figuras 5E e 5F são mostrados os valores diários de tensão, utilizando os SA e TS, respectivamente, para o tratamento de $60 \mathrm{kPa}$. Na Figura 6 é mostrado o número de irrigações registradas nas diferentes tensões, utilizando diferentes métodos.

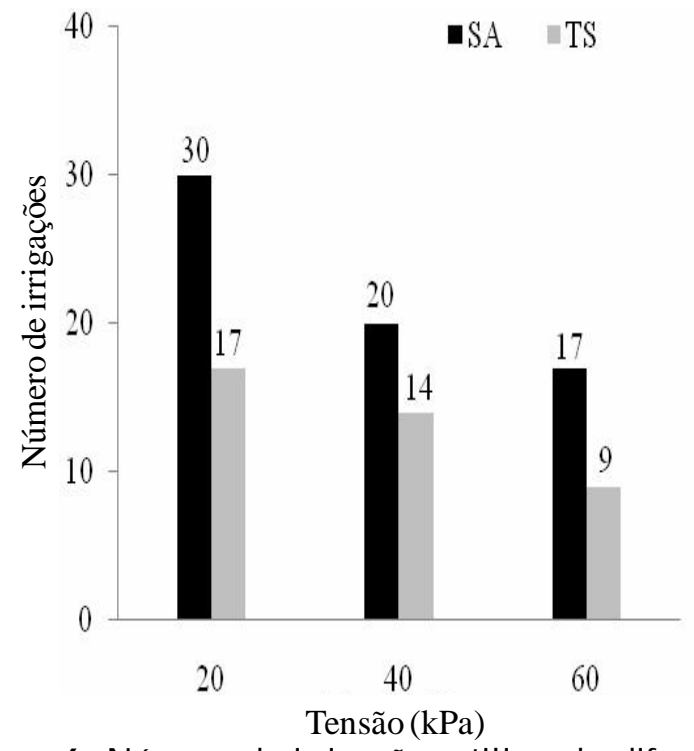

Figura 6. Número de irrigações utilizando diferentes métodose tensões

Observa-se que, independente da tensão, o manejo da irrigação utilizando o $\mathrm{SA}$, apresentou maior número de irrigações.

\section{ConclusõEs}

1. Independente do método empregado, a maior produção do feijoeiro foi no tratamento submetido a tensões próximas a $39 \mathrm{kPa}$.
2. Os sensores alternativos avaliados foram mais susceptíveis às variações de umidade do solo, em relação aos tensiômetros.

3. Os valores de capacitância tiveram maior variação na tensão de $20 \mathrm{kPa}$.

4. O número de irrigações e o volume de água aplicado foram maiores no método empregando-se os sensores alternativos, implicando numa eficiência menor do uso da água.

\section{LITERATURA CITADA}

Anderson, A. B. C. A method of determining soil-moisture content based on the variation of the electrical capacitance of soil, at a low frequency, with moisture content. Soil Science, v.56, p.29-41, 1943.

Anderson, A. B. C.; Edlefsen, N. E. The electrical capacity of the 2-electrode plaster of paris block as an indicator of soilmoisture content. Soil Science, v.54, p.35-46, 1942.

Benedetto, A. Water content evaluation in unsaturated soil using GPR signal analysis in the frequency domain. Journal of Applied Geophysics, v.71, p.26-35, 2010.

Celinski, V. G.; Zimback, C. R. Avaliação de um sensor de capacitância elétrica e sua correlação com atributos do solo. Revista Energia na Agricultura, v.25, p.157-170, 2010.

CFSEMG - Comissão de Fertilidade do Solo do Estado de Minas Gerais. Recomendações para o uso de corretivos e fertilizantes em Minas Gerais. In: Ribeiro, A. C.; Guimarães, P. T. G.; Alvarez V., V. H. (ed.), 5.ed. Lavras: CFSEMG, 1999. 359p.

Chang, Z. Y.; Iliev, B. P.; Groot, J. F.; Meijer, G. C. M. Extending the limits of a capacitive soil-water-content measurement. Ieee Transactions on Instrumentation and Measurement, v.56, p.2240-2244, 2007.

Conklin, R. A. Introduction to soil chemistry: Analysis and instrumentation. Hoboken,: Wiley - Interscience, 2005. $218 \mathrm{p}$.

Cruz, T. M. L.; Texeira, A. S.; Canafístula, F. J. F.; Santos, C. C.; Oliveira, A. D. S.; Daher, S. Avaliação de sensor capacitivo para o monitoramento do teor de água do solo. Engenharia Agrícola, v.30, p. 33-45, 2010.

Dantas, A. A. A.; Carvalho, L. G.; Ferreira, E. Classificação e tendências climáticas em Lavras, MG. Ciência e Agrotecnologia, v.31, p.1862-1866, 2007.

EMBRAPA - Empresa Brasileira de Pesquisa Agropecuária. Centro Nacional de Pesquisas de Solos. Sistema brasileiro de classificação de solos. 2.ed. Rio de Janeiro: Embrapa Solos, 2006. 306p.

Frangi, J. P.; Richard, D. C.; Chavanne, X.; Bexi, I.; Sagnard, F.; Guilbert, V. New in situ techniques for the estimation of the dielectric properties and moisture content of soils. Comptes Rendus Geoscience, v.341, p.831-845, 2009.

Ferreira, D. F. Análises estatíticas por meio do Sisvar para Windows versão 4.0 In: Reunião Anual da Região Brasileira da Sociedade Internacional de Biometria, 45., 2000, São Carlos, SP. Anais... São Carlos: Sociedade Internacional de Biometria, 2000. p.255-258. 
Freitas, W. A.; Carvalho, J. A.; Bilibio, C.; Lacerda, W. S. Avaliação de dispositivo, de baixo custo, para determinação de potencial matricial e umidade do solo. In: Congresso Brasileiro De Engenharia Agrícola, 38, 2009, Petrolina. Anais... Petrolina: CONBEA, 2009. CD-Rom

Genuchten, M. T. H. van. Aclosed-form equation for predicing the hydraulic conductivity for unsatured soils. Soil Science Society American Journal, v. 4, p 892-898, 1980.

Guimarães, C. M.; Stone, L. F.; Brunini, O. Adaptação do feijoeiro comum (Phaseolus vulgaris L.) à seca. Revista Brasileira de Engenharia Agrícola e Ambiental, v.10, p.70-75, 2006.

Kizito, F.; Campbell, C. S.; Campbell, G. S.; Cobos, D. R.; Teare, B. L.; Carter, B.; Hopmans, J. W. Frequency, electrical conductivity and temperature analysis of a low-cost capacitance soil moisture sensor. Journal of Hydrology, v. 352, p. 367-378, 2008.

Kumhála, F.; Prosek, V.; Blahovec, J. Capacitive throughput sensor for sugar beets and potatoes. Biosystems Engineering, v.102, p.36-43, 2008.

Lopes, A. da S.; Oliveira, G. Q.; Filho, S. N. S.; Goes, R. J.; Camacho, M. A. Manejo de irrigação e nitrogênio no feijoeiro comum cultivado em sistema plantio direto. Revista Ciência Agronômica, v.42, p.51-56, 2011.

Monsen-Nia, M.; Amiri, H.; Jazi, B. Dielectric constants of water, methanol, ethanol, butanol and acetone: measurement and computational study. Journal of Solution Chemistry, v.39, p.701-708, 2010.
Monteiro, J. B. A. (Org.). Agrometeorologia dos cultivos: o fator meteorológico na produção agrícola. 1.ed. Brasília: Instituto Nacional de Meteorologia, 2009. 530p.

Paula Júnior, T. J.; Vieira, R. F.; Teixeira, H.; Coelho, R. R.; Carneiro, J. E. S.; Andrade, M. J. B.; Resende, A. M. Informações técnicas para o cultivo de feijoeiro-comum na região central brasileira: 2007-2009. Viçosa: EPAMIG, 2008. 180p.

Persson, M.; Wraith, J. M.; Dahlin, T.A small-scale matric potential sensor based on time domain reflectometry. Soil Science Society of America Journal, v.70, p.533-536, 2006.

Plater, C. V. de. A portable capacitance: Type soil moisture meter. Soil Science Society American Journal, v.80, p.391-396, 1955.

Queiroz, T. M. de. Avaliação de sistema alternativo de automação da irrigação na cultura do feijoeiro (Phaseolus vulgaris L.). Lavras: UFLA, 2004. 120p. Dissertação Mestrado

Rusiniak, L. Spontaneous polarization of water in porous structure of a solid body. Geophysical Journal International, v.148, p.313-319, 2002.

Sant'Ana, E. V. P.; Santos, A. B.; Silveira, P. M. Eficiência de uso de nitrogênio em cobertura pelo feijoeiro irrigado. Revista Brasileira de Engenharia Agrícola e Ambiental, v.15, p.458-462, 2011.

Williams, G. The dielectrics discussion group (UK) and dielectrics society platforms for dielectrics research. IEEE Transactions on Dielectrics and Electrical Insulation, v.18, p.570-578, 2011. 